\title{
Biochemical and Hematological Effects of Food Flavoring Furfural in Male Albino Rats
}

\author{
Tarek M. Ali ${ }^{1}$, Osama M. Ahmed ${ }^{2}$, Rasha R. Ahmed ${ }^{3}$.
} ${ }^{1}$ Physiology Department, Faculty of Medicine, ${ }^{2}$ Physiology Division, and ${ }^{3}$ Histology division, Zoology Department, Faculty of Science, Beni-Suef University

\begin{abstract}
Objectives: The present study aims to assess the effect of furfural, an aldehyde commonly used as food flavor in many foods, on the hematological and biochemical aspects in male albino rat. Materials and methods: The experimental animals used in the study were divided into four groups. The control group was given a daily volume of the vehicle dimethyl sulfoxide (DMSO) whereas the other three groups were orally administered furfural for 8 weeks in a dose of $3.18,6.36$ and $12.72 \mathrm{mg} / \mathrm{kg}$ body weight respectively. Blood samples were obtained for complete blood count (CBC), liver and kidney function tests. Serum $\alpha$-fetoprotein $(A F P)$ and carbohydrate antigen ( $C A_{19.9}$ were also measured. Results: Obtained results revealed a decrease in red blood corpuscles (RBCS) count, packed cell volume (PCV) and hemoglobin content and an increase in mean cell volume (MCV) and mean cell hemoglobin content $(M C H)$ in rats received furfural in a dose dependent manner. The white blood cells (WBC) count was decreased specially esinophils percentage was depleted in the low and medium doses groups. Furfural elevated serum liver enzymes activities and total bilirubin concentration. The serum total proteins and albumin levels were increased in medium and high doses groups, while globulin level, was elevated only in low dose group. Furfural elevated serum urea level but did not affect serum AFP and $C A_{19.9}$ levels in all groups. Conclusions: In conclusion, furfural administration to rats induces a sort of macrocytic anemia, leucopenia, esinopenia and elevated liver enzymes and urea in rats in a dose related manner.
\end{abstract}

\section{INTRODUCTION}

Furfural (furan-2-carboxyaldehyde, $\mathrm{C}_{4} \mathrm{H}_{3} \mathrm{OCHO}$ ) was first extracted in 1840 through the bran distillation (Latin: furfur) by diluted sulfuric acid ${ }^{(1)}$. Furfural is a colorless liquid with pleasant aroma and it is partially soluble in water. It has a significant use as a selective solvent of mineral oil products, in chemistry, in the rubber industry, plastic surgery and polymer industry ${ }^{(2)}$. It is also present in orange juice, brandy ${ }^{(3)}$ and Japanese sake $e^{(4)}$. In the organism, furfural occurs by pentose dehydration ${ }^{(5)}$ In Europe, furfural is used as a flavor in foods such as baked goods, frozen dairies, meat products, candy, puddings, beverages, and gravies ${ }^{(6)}$. Furfural is a carcinogen classified in EU as a Category 3 carcinogen. In mice, it increased the incidence of hepatocellular adenomas 
and carcinomas in males and of hepatocellular adenomas and fore stomach papillomas in females ${ }^{(7)}$. Furfural is eliminated slowly and in unchanged form through the kidneys and lungs. The liver oxidizes it into pyromucic acid $\left(\mathrm{C}_{4} \mathrm{H}_{3} \mathrm{OCOOH}\right)$ which is toxic to hepatocytes, but it is conjugated with glycine and mostly excreted in urine ${ }^{(8,9)}$. Furfural is unsaturated cyclic aldehyde, it is a known hepatotoxic substance ${ }^{(\mathbf{1 0})}$, and it changes the activity of some enzymes in the liver in acute ${ }^{(11)}$ and chronic $^{(12)}$ experiments. Furfural may cause morphologic changes in the liver ${ }^{(\mathbf{1 3})}$ that in the acute experiment are manifested in diffuse necrosis associated with regeneration of hepatocytes and terminate within a few days with liver recovery. It is presumed that liver damage is induced by oxidation of furfural. In the chronic experiment, furfural induces cirrhotic changes $^{(13)}$ associated with pseudolobule formation, enlargement of the portal area, and destruction of the border plaque. In the liver parenchyma, a marked bridging necrosis and hydropic degeneration of hepatocytes develop. Simultaneously, various degrees of liver insufficiency may become evident ${ }^{(\mathbf{1 4}, 15)}$. Liver transaminases, AST and ALT are important markers of liver damage and are routinely done in modern laboratories. Also, $\gamma$-GT and alkaline phosphatase (ALP) are increased in cases where there is damage to liver parenchyma under the influence of many harmful substances which are detoxified in the liver every day ${ }^{(\mathbf{1 6})}$.

The aim of the current study is to clarify the hazards of oral furfural use in different doses on the hematological and biochemical parameters including liver and kidney functions tests in male albino rats

\section{MATERIALS \& METHODS}

Experimental animals:

Male albino rats (Rattus norvegicus) weighing 140-160 g were obtained from the animal house of Institute of Ophthalmology, Giza, Egypt. They were housed in plastic cages with good aerated covers at normal temperature and normal daily light-dark cycle. The animals were fed standard diet pellets and tap water ad libitum. They were kept under observation for 2 weeks to exclude any intercurrent infection and for proper acclimatization. All animal procedures were in accordance with the recommendations for the proper care and use of laboratory animals of Canadian Council on Animal Care ${ }_{(\mathrm{CCAC})}{ }^{(17)}$.

Furfural:

2-Furancaboxyaldehyde, known commercially as furfural, was obtained from Aldrich company, Gillingham, England. The different doses of furfural-dissolved in dimethyl sulfoxide (DMSO)-were given daily to rats by gastric intubation between 8-10 AM. Three doses were chosen on the basis of the oral LD50 value which was previously determined by Jenner et al. ${ }^{(18)}$ and equals $127 \mathrm{mg} / \mathrm{Kg}$ body weight.

\section{Animal Grouping and Experimental design:}

The experimental animals used in the present study were divided into four groups. One group was kept as control and was given a daily 
respective volume of the vehicle (DMSO) whereas the other three groups were orally administered $1 / 40$, $1 / 20$ and $1 / 10$ of LD50 corresponding to approximately to $3.18,6.36$ and $12.72 \mathrm{mg} / \mathrm{kg}$ body weight respectively.

All groups were treated for 8 weeks. At the end of that period, rats were sacrificed and blood samples were obtained from jugular vein in two tubes, one containing ethylene diamine tetraacetic acid solution $15 \%$ (50 $\mu \mathrm{l}$ EDTA $/ 5 \mathrm{ml}$ blood) for determination of blood indices and the other without EDTA. After blood coagulation in the $2^{\text {nd }}$ tube, blood was centrifuged at $3000 \mathrm{rpm}$ for 15 minutes. Serum was obtained from each tube and kept at $-30^{\circ} \mathrm{C}$ till used for biochemical determination.

\section{Hematological Examination:}

Counting of the red blood corpuscles (RBCs) and white blood cells (WBCs) was carried out by Neubauer slide, using saline $(0.9 \%$ $\mathrm{NaCl})^{(\mathbf{1 9 )}}$ and Turk's fluid $^{(\mathbf{2 0})}$ respectively. The hematocrit value was determined according to Dacie and Lewis ${ }^{(21)}$ and the hemoglobin content was measured as described by Lucky $^{(22)}$. Also, mean cell volume (MCV), mean cell hemoglobin content, and mean cell hemoglobin concentration percent (MCHC) were calculated according to Dacie and Lewis $^{\text {(21) }}$. Blood film was prepared, fixed in methanol and then stained with Giemsa stain according to the methods of Houwen ${ }^{(23)}$.

Biochemical Examinations:

Serum alanine aminotransferase (ALT) and aspartate aminotransferase (AST) activities were determined according to the method of
Bergmeyer et al. ${ }^{(24)}$, respectively, using reagent kits purchased from Spinreact company (Spain). Serum gamma-glutamyl transferase $(\gamma-\mathrm{GT})$ activity was determined according to Beleta and Gella ${ }^{(25)}$. Serum alkaline phosphatase (AP) activity was assayed according to the method of Belfield and Goldberg ${ }^{(26)}$ using reagent kit obtained from BioMerieux Chemical company (France). Serum lactate dehydrogenase activity was estimated by kits obtained from Stanbio Laboratories (USA) according to the method of Bühl and Jackson ${ }^{(27)}$. Total bilirubin concentration was determined according to the method of Jendrassik and Grap ${ }^{(28)}$ using reagent kits purchased from Diamond Diagnostics Chemical Company, Egypt.

Serum total protein and serum albumin levels were estimated according to Henry ${ }^{(29)}$ and Doumas et al. ${ }^{(30)}$ using reagent kits purchased from Diamond Diagnostics Chemical company, Egypt. Serum globulin level was calculated by subtracting albumin level form total protein concentration. In addition, the ratio of albumin to globulin was calculated.

Serum $\alpha$-fetoprotein (AFP) concentration was determined by a radioimmunoassay kit [Double antibody kit, Diagnostic Products Corporation (DPC), USA] according to the methods of Waldmann and Mclntire $^{(31)}$ and Wepsic ${ }^{(32)}$. Serum carbohydrate antigen $\left(\mathrm{CA}_{19.9}\right.$ was estimated by an immunoradiometric assay kit (Coat-A-Count GI-MA IRMA, DPC, USA) according to the method of Ferbourg et al. ${ }^{(33)}$. Both AFP and $\mathrm{CA}_{19.9}$ concentrations were measured in the Radioactive Isotope 
Unit, National Research Center, Dokki, Cairo, Egypt.

Serum urea and creatinine concentrations were measured based respectively on the methods of Patton and Crouch ${ }^{(34)}$ and Henry ${ }^{(35)}$ using reagent kits produced by Diamond Diagnostics (Egypt). Serum uric acid level was determined according to the method of Barham and Trinder ${ }^{(36)}$ using reagent kits obtained from Spinreact Company (Spain).

Statistical analysis: The data were analyzed using the one way analysis of variance (ANOVA) ${ }^{(37)}$ followed by LSD analysis to compare various groups with each other. Results were expressed as mean \pm standard error (SE). Values of $\mathrm{P}>0.05$ were considered statistically nonsignificantly different, while values of $\mathrm{P}<0.05$ and $\mathrm{P}<0.01$ were significantly and highly significantly different respectively. F-probability expresses the general effect between groups.

\section{RESULTS}

\section{Hematological effects:}

The administration of furfural to albino rats for 8 weeks produced significant decrease of red blood corpuscles count, hematocrit (PCV) and hemoglobin content at the medium and high doses (6.36 and $12.72 \mathrm{mg} / \mathrm{kg}$ body weight) in a dose dependent manner. However, these parameters were not significantly affected at low dose $(3.18 \mathrm{mg} / \mathrm{kg}$ body weight). In contrast, mean cell volume (MCV) of red blood corpuscles as well as mean cell hemoglobin $(\mathrm{MCH})$ were highly significantly $(\mathrm{P}<0.01$; LSD) increased as a result of administration of medium and high concentration of furfural in a dose dependent manner. Mean cell hemoglobin concentration (MCHC) was not significantly $(\mathrm{P}>0.01$; LSD) affected as a result of all tested doses of furfural. ANOVA results revealed that the effect between groups on $\mathrm{RBC}$ count, $\mathrm{MCH}$ and MCV was very highly significant $(\mathrm{P}<0.001$; F-prob.) while the effect on hematocrit, $\mathrm{Hb}$ and $\mathrm{MCHC}$ was only significant $(\mathrm{P}<0.05$; F-prob.) (Table1).

The white blood cells (WBC) or leucocytes count was significantly decreased due to furfural administration for 8 weeks; the percentage decreases were 26.804, 41.842 and $40.689 \%$ respectively as a result of low, medium and high doses of furfural. Both lymphocytes and monocytes percentages were detectably decreased in furfuraladministered animals although these changes were not significant. Esinophils percentage was highly significantly depleted as a result of low and medium doses administration. Basophils percentage was also not significantly affected. ANOVA results revealed that while the effect between groups on WBC count and neutrophil percent was highly significant $(\mathrm{P}<0.01 ;$ F-prob. $)$, the effect on esinophil percent was very highly significant $\quad(\mathrm{P}<0.001 ; \quad$ F-prob. $)$ (Table2).

\section{Biochemical Effects:}

Serum ALT, AST, $\gamma$ GT, ALP and $\mathrm{LDH}$ activities as well as total bilirubin concentration were remarkably increased as a result of furfural administration. The LDH activity and total bilirubin concentration were increased in a dose 
dependent manner. While the low dose produced the most potent effect on AST activity, the high dose seemed to be the most effective on ALT, $\gamma \mathrm{GT}$, ALP and LDH activities and total bilirubin concentration. One-way ANOVA indicated that the general effect was significant $(\mathrm{P}<0.05$; Fprob.) on LDH activity, highly significant $(\mathrm{P}<0.01)$ on $\gamma \mathrm{GT}$ activity and total bilirubin concentration and very highly significant $(\mathrm{P}<0.001)$ on ALT, AST and ALP activities (Table $3)$.

The serum total protein and albumin levels were significantly $(\mathrm{P}<0.05 ; \quad$ LSD $)$ and highly significantly $(\mathrm{P}<0.01$; LSD) increased as a result of medium and high doses of furfural, respectively (figure 1). Serum globulin level, on the other hand, was significantly elevated due only to low dose. $\mathrm{A} / \mathrm{G}$ ratio was significantly $(\mathrm{P}<0.01$; LSD) increased as a result of low and high dose of furfural (figure 2). Serum AFP and CA ${ }_{19.9}$ levels was non-significantly affected as a result of all tested doses of furfural (figure 3) as indicated by one-way ANOVA (highly significantly $(\mathrm{P}<0.01 ;$ F-prob. $)$ and LSD test $(\mathrm{P}>0.05)$

Serum creatinine and uric acid levels were not significantly changed in furfural administered rats. Serum urea level, on the other hand, was highly significantly increased as a result of all tested doses of furfural; the percentage increases were 32.665 , 56.903 and 42.820 respectively due to low, medium and high doses respectively (Table 4). One-way ANOVA revealed that the effect between groups on urea level and $\mathrm{A} / \mathrm{G}$ ratio was highly significant and significant respectively. 
Table 1: The effect of furfural on hematological indices of rats in control, low ,moderate and high dose groups

\begin{tabular}{|c|c|c|c|c|c|c|c|c|c|c|c|c|c|}
\hline \multicolumn{2}{|c|}{ Group } & $\begin{array}{c}\text { Total } \\
\text { RBCs } \\
\text { count } \\
\times 10^{6} / \mu \mathrm{I} \\
\end{array}$ & $\%$ & $\begin{array}{c}\text { PCV } \\
\%\end{array}$ & $\%$ & $\mathrm{Hb}(\mathrm{g} / \mathrm{dl})$ & $\%$ & MCH Pg & $\%$ & $\underset{\left(\mu^{3}\right)}{\operatorname{MCV}(F I}$ & $\%$ & $\underset{\%}{\mathrm{MCHC}}$ & $\%$ \\
\hline \multicolumn{2}{|c|}{ Control } & $\begin{array}{l}5.7322^{ \pm} \\
0.122^{\mathrm{a}}\end{array}$ & & $\begin{array}{c}46.481 \pm \\
0.229^{\mathrm{a}}\end{array}$ & & $\begin{array}{c}13.549 \pm \\
0.292^{\mathrm{a}} \\
\end{array}$ & & $\begin{array}{c}23.549 \pm \\
0.845^{\mathrm{c}}\end{array}$ & & $\begin{array}{c}81.430 \pm \\
1.842^{\mathrm{c}}\end{array}$ & & $\begin{array}{c}29.287 \pm \\
1.031^{\mathrm{a}}\end{array}$ & \\
\hline \multirow{3}{*}{ } & $\begin{array}{l}\text { Low } \\
\text { dose }\end{array}$ & $\begin{array}{l}6.036 \pm \\
0.497^{\text {a }}\end{array}$ & $\begin{array}{l}m \\
\tilde{m} \\
i \\
+ \\
+\end{array}$ & $\begin{array}{c}45.130 \pm \\
0.594^{\mathrm{ab}}\end{array}$ & 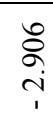 & $\begin{array}{c}12.169 \pm \\
0.384^{\mathrm{b}}\end{array}$ & \begin{tabular}{l}
\multirow{0}{0}{} \\
$\infty$ \\
$i$
\end{tabular} & $\begin{array}{r}20.902 \text { 士 } \\
1.898^{\mathrm{c}}\end{array}$ & 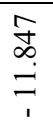 & $\begin{array}{c}82.311 \pm \\
7.252^{\mathrm{bc}}\end{array}$ & $\begin{array}{l}\tilde{O} \\
\stackrel{0}{0} \\
+ \\
+\end{array}$ & $\begin{array}{c}27.224 \pm \\
1.074^{\mathrm{a}}\end{array}$ & 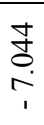 \\
\hline & $\begin{array}{l}\text { Moderate } \\
\text { dose }\end{array}$ & $\begin{array}{c}4.6411^{ \pm} \\
0282^{b}\end{array}$ & $\begin{array}{l}\infty \\
\sim \\
\sim \\
\sim \\
\end{array}$ & $\begin{array}{l}43.604 \pm \\
1.806^{\mathrm{bc}}\end{array}$ & $\begin{array}{l}\infty \\
\overrightarrow{0} \\
1\end{array}$ & $\begin{array}{c}12.587 \pm \\
0.289^{\mathrm{ab}}\end{array}$ & $\underset{\substack{0 \\
\hdashline}}{\stackrel{0}{1}}$ & $\begin{array}{c}28.310^{ \pm} \\
1.149^{\mathrm{b}}\end{array}$ & 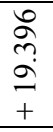 & $\begin{array}{c}98.635 \pm \\
6.727^{\mathrm{b}}\end{array}$ & $\begin{array}{l}\infty \\
\stackrel{\infty}{I} \\
\stackrel{\vec{N}}{+} \\
+\end{array}$ & 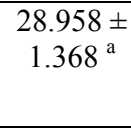 & 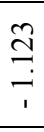 \\
\hline & high dose & $\begin{array}{l}2.702^{ \pm} \\
0.141^{\mathrm{c}}\end{array}$ & $\begin{array}{l}\vec{b} \\
\infty \\
i \\
i \\
1\end{array}$ & $\begin{array}{c}41.574 \text { 土 } \\
0.123^{\mathrm{c}}\end{array}$ & $\begin{array}{l}n \\
n \\
0 \\
1\end{array}$ & $\begin{array}{c}11.692 \text { 士 } \\
0.694^{\mathrm{b}}\end{array}$ & $\begin{array}{l}\stackrel{r}{?} \\
\stackrel{2}{r}\end{array}$ & $\begin{array}{c}43.837 \pm \\
1.695^{\mathrm{a}}\end{array}$ & $\begin{array}{l}\infty \\
\infty \\
\infty \\
+ \\
+\end{array}$ & $\begin{array}{c}156.611 \pm \\
4.887^{\mathrm{a}}\end{array}$ & $\begin{array}{l}\text { ते } \\
\text { } \\
\text { Lे } \\
+\end{array}$ & $\begin{array}{c}28.176 \pm \\
1.435^{\mathrm{a}}\end{array}$ & $\begin{array}{l}\stackrel{m}{q} \\
\stackrel{r}{i}\end{array}$ \\
\hline \multicolumn{2}{|c|}{ F-probability } & \multicolumn{2}{|c|}{$\mathrm{P}<0.01$} & \multicolumn{2}{|c|}{$\mathrm{P}<0.05$} & \multicolumn{2}{|c|}{$\mathrm{P}<0.05$} & \multicolumn{2}{|c|}{$\mathrm{P}<0.001$} & \multicolumn{2}{|c|}{$\mathrm{P}<0.001$} & \multicolumn{2}{|c|}{$P>0.05$} \\
\hline \multicolumn{2}{|c|}{ LSD at $5 \%$} & \multicolumn{2}{|c|}{0.887} & \multicolumn{2}{|l|}{2.831} & \multicolumn{2}{|c|}{1.318} & \multicolumn{2}{|c|}{4.303} & \multicolumn{2}{|c|}{16.500} & \multicolumn{2}{|l|}{-} \\
\hline \multicolumn{2}{|c|}{ LSD at $1 \%$} & \multicolumn{2}{|c|}{1.210} & \multicolumn{2}{|l|}{3.861} & \multicolumn{2}{|c|}{1.798} & \multicolumn{2}{|c|}{5.869} & \multicolumn{2}{|c|}{22.503} & \multicolumn{2}{|l|}{ - } \\
\hline
\end{tabular}

- Data are expressed as Mean \pm SE. Number of animals in each group is six.

- Means, which share the same superscript symbol(s), are not significantly different. 
Table 2: The effect of furfural on total and differential WBCs count ( $\%$ of total ) in control, low ,moderate and high dose groups

\begin{tabular}{|c|c|c|c|c|c|c|c|c|c|c|c|c|c|}
\hline \multirow{2}{*}{\multicolumn{2}{|c|}{ Group }} & \multirow{2}{*}{$\begin{array}{c}\text { Total WBCs } \\
\text { count } x \\
10^{3} / \mu \mathrm{l} \\
\end{array}$} & \multirow[t]{2}{*}{$\%$} & \multicolumn{10}{|c|}{ Differential WBCs count ( \% o total ) } \\
\hline & & & & $\begin{array}{c}\text { Lympho } \\
\text { cytes }\end{array}$ & $\%$ & $\begin{array}{c}\text { Neutrophil } \\
\text { (segmented) }\end{array}$ & $\%$ & $\begin{array}{c}\text { Mono } \\
\text { cytes }\end{array}$ & $\%$ & Eosinophil & $\%$ & $\begin{array}{c}\text { Baso } \\
\text { phil }\end{array}$ & $\%$ \\
\hline \multicolumn{2}{|c|}{ Control } & $\begin{array}{l}7.980 \pm \\
1.009^{\mathrm{a}}\end{array}$ & & $\begin{array}{l}75.5 \pm \\
2.473^{\mathrm{a}}\end{array}$ & & $\begin{array}{c}17.333 \pm \\
1.202^{\mathrm{c}}\end{array}$ & & $\begin{array}{l}1.666 \pm \\
0.333^{\mathrm{a}}\end{array}$ & & $\begin{array}{l}7.000 \pm \\
0.683^{\text {a }}\end{array}$ & & 0 & \\
\hline \multirow{3}{*}{ 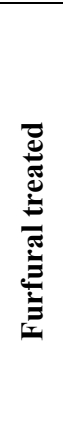 } & $\begin{array}{l}\text { Low } \\
\text { dose }\end{array}$ & $\begin{array}{l}5.841 \pm \\
0.578^{\mathrm{b}}\end{array}$ & $\begin{array}{l}\stackrel{+}{ } \\
\infty \\
\stackrel{+}{+} \\
1\end{array}$ & $\begin{array}{l}70.00 \pm \\
5.360^{\mathrm{a}}\end{array}$ & $\stackrel{n}{\infty}$ & $\begin{array}{c}26.000 \pm \\
1.341^{\mathrm{a}}\end{array}$ & 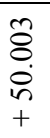 & $\begin{array}{l}0.8333^{ \pm} \\
0.477^{\text {a }}\end{array}$ & in & $\begin{array}{l}3.000 \pm \\
0.730^{\mathrm{b}}\end{array}$ & $\underset{i}{\stackrel{m}{+}}$ & 0 & \\
\hline & $\begin{array}{l}\text { Moderate } \\
\text { dose }\end{array}$ & $\begin{array}{l}4.641 \pm \\
0.393^{\mathrm{b}}\end{array}$ & 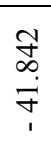 & $\begin{array}{c}74.833 \pm \\
2.856^{\mathrm{a}}\end{array}$ & $\begin{array}{l}0 \\
\infty \\
\infty \\
0 \\
1\end{array}$ & $\begin{array}{c}21.166 \pm \\
1.351^{\mathrm{bc}}\end{array}$ & 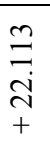 & $\begin{array}{l}1.000 \pm \\
0.258^{\mathrm{a}}\end{array}$ & 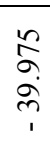 & $\begin{array}{l}3.666 \pm \\
0.333^{\mathrm{a}}\end{array}$ & 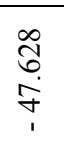 & 0 & \\
\hline & $\begin{array}{l}\text { high } \\
\text { dose }\end{array}$ & $\begin{array}{l}4.733^{ \pm} \\
0.553^{\mathrm{b}}\end{array}$ & $\begin{array}{l}\infty \\
\infty \\
\dot{0} \\
\dot{+} \\
1 \\
1\end{array}$ & $\begin{array}{c}69.333 \pm \\
4.402^{\mathrm{a}}\end{array}$ & $\begin{array}{l}\infty \\
\infty \\
\infty \\
1\end{array}$ & $\begin{array}{l}22.000 \pm \\
5.190^{\mathrm{ab}}\end{array}$ & 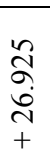 & $\begin{array}{l}0.500 \pm \\
0.498^{a}\end{array}$ & 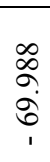 & $\begin{array}{l}8.000 \pm \\
0.806^{\mathrm{a}}\end{array}$ & \begin{tabular}{l}
8 \\
$\infty$ \\
\cline { 1 - 1 } \\
\pm \\
+ \\
+
\end{tabular} & 0 & \\
\hline \multicolumn{2}{|c|}{ F-probability } & \multicolumn{2}{|l|}{$\mathrm{P}<0.01$} & \multicolumn{2}{|c|}{$\mathrm{P}>0.05$} & \multicolumn{2}{|l|}{$\mathrm{P}<0.01$} & \multicolumn{2}{|c|}{$\mathrm{P}>0.05$} & \multicolumn{2}{|c|}{$\mathrm{P}<0.001$} & \multicolumn{2}{|c|}{$\mathrm{P}>0.05$} \\
\hline \multicolumn{2}{|c|}{ LSD at $5 \%$} & \multicolumn{2}{|l|}{1.986} & \multicolumn{2}{|l|}{-} & \multicolumn{2}{|l|}{4.634} & \multicolumn{2}{|l|}{-} & \multicolumn{2}{|l|}{1.957} & \multicolumn{2}{|c|}{-} \\
\hline \multicolumn{2}{|c|}{ LSD at $1 \%$} & \multicolumn{2}{|l|}{2.708} & \multicolumn{2}{|l|}{-} & \multicolumn{2}{|l|}{6.321} & - & & \multicolumn{2}{|l|}{2.669} & \multicolumn{2}{|c|}{-} \\
\hline
\end{tabular}

- Data are expressed as Mean \pm SE. Number of animals in each group is six.

- Means, which share the same superscript symbol(s), are not significantly different.

- Stuff cells are zero in all groups 
Table 3: The effect of furfural on liver enzymes and total bilirubin in control , low ,moderate and high dose groups

\begin{tabular}{|c|c|c|c|c|c|c|c|c|c|c|c|c|c|}
\hline \multicolumn{2}{|c|}{ Group } & $\begin{array}{l}\text { ALT } \\
\text { (U/L) }\end{array}$ & $\%$ & $\begin{array}{c}\text { AST } \\
(\mathbf{U} / \mathbf{L})\end{array}$ & $\%$ & $\begin{array}{l}\gamma \mathbf{G T} \\
(\mathbf{U} / \mathbf{L})\end{array}$ & $\%$ & $\begin{array}{l}\text { ALP } \\
\text { (U/L) }\end{array}$ & $\%$ & $\begin{array}{l}\text { LDH } \\
\text { (U/dl) }\end{array}$ & $\%$ & $\begin{array}{c}\text { Total } \\
\text { bilirubin } \\
\text { (mg/dl) }\end{array}$ & $\%$ \\
\hline \multicolumn{2}{|c|}{ Control } & $\begin{array}{c}41.300 \pm \\
3.309^{\mathrm{b}}\end{array}$ & & $\begin{array}{c}154.00 \pm \\
3.443^{\mathrm{c}}\end{array}$ & & $\begin{array}{c}15.333^{ \pm} \\
0.882^{\mathrm{c}}\end{array}$ & & $\begin{array}{c}203.100 \pm \\
1.050^{\mathrm{b}}\end{array}$ & & $\begin{array}{l}90.383 \pm \\
8.490 \mathrm{~b}\end{array}$ & & $\begin{array}{c}0.566 \pm \\
0.042 \mathrm{~b}^{\mathrm{a}}\end{array}$ & \\
\hline \multirow{3}{*}{ 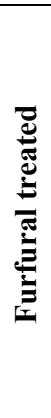 } & $\begin{array}{l}\text { Low } \\
\text { dose }\end{array}$ & $\begin{array}{c}64.681 \pm \\
4.246^{\mathrm{a}}\end{array}$ & $\begin{array}{l}\text { ô } \\
\text { ñ? } \\
+ \\
+\end{array}$ & $\begin{array}{c}248.600 \pm 20 \\
.907^{\mathrm{a}}\end{array}$ & $\begin{array}{l}\stackrel{1}{\sigma} \\
\stackrel{0}{0} \\
\stackrel{+}{+}\end{array}$ & $\begin{array}{c}19.600 \pm \\
0.219^{\mathrm{b}}\end{array}$ & $\begin{array}{l}\stackrel{\text { సे }}{\infty} \\
\stackrel{\sim}{+}\end{array}$ & $\begin{array}{c}332.000 \pm \\
7.399^{\mathrm{a}}\end{array}$ & $\begin{array}{l}0 \\
0 \\
+ \\
\dot{0} \\
+ \\
+\end{array}$ & $\begin{array}{c}93.906 \pm \\
6.389^{\mathrm{b}}\end{array}$ & $\begin{array}{l}\infty \\
\infty \\
\dot{m} \\
+\end{array}$ & $\begin{array}{l}0.675 \pm \\
0.054^{\mathrm{b}}\end{array}$ & $\begin{array}{l}\hat{n} \\
\stackrel{n}{2} \\
+\end{array}$ \\
\hline & $\begin{array}{l}\text { Moderate } \\
\text { dose }\end{array}$ & $\begin{array}{c}63.375 \pm \\
2.897^{\mathrm{a}}\end{array}$ & $\begin{array}{l}\stackrel{n}{n} \\
\stackrel{5}{0} \\
\stackrel{n}{n} \\
+\end{array}$ & $\begin{array}{c}207.400 \pm \\
7.779^{\mathrm{b}}\end{array}$ & $\begin{array}{l}n \\
\hat{6} \\
+ \\
+ \\
+\end{array}$ & $\begin{array}{l}19.012 \pm \\
1.258^{\mathrm{bc}}\end{array}$ & \begin{tabular}{l}
\multirow{+}{\sigma}{} \\
ते \\
+
\end{tabular} & $\begin{array}{c}329.066 \pm \\
11.078^{\mathrm{a}}\end{array}$ & 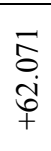 & $\begin{array}{c}100.150 \pm \\
0.980^{\mathrm{b}}\end{array}$ & $\begin{array}{l}\stackrel{0}{0} \\
\infty \\
0 \\
\\
+\end{array}$ & $\begin{array}{l}0.883 \pm \\
0.079^{\mathrm{a}}\end{array}$ & $\begin{array}{l}\hat{8} \\
0 \\
\dot{0} \\
+\end{array}$ \\
\hline & high dose & $\begin{array}{c}66.425 \pm \\
5.571^{\mathrm{a}}\end{array}$ & $\begin{array}{l}n \\
\infty \\
0 \\
0 \\
0 \\
+\end{array}$ & $\begin{array}{c}201.400 \pm 3 . \\
443^{\mathrm{b}}\end{array}$ & 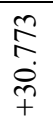 & $\begin{array}{c}24.050 \pm \\
2.393^{\mathrm{a}}\end{array}$ & $\begin{array}{l}\bar{n} \\
\infty \\
0 \\
\infty \\
+\end{array}$ & $\begin{array}{c}388.366 \pm \\
37.933^{\text {a }}\end{array}$ & $\begin{array}{l}n \\
n \\
\infty \\
\infty \\
+\end{array}$ & $\begin{array}{c}117.566 \pm \\
3.692^{\mathrm{a}}\end{array}$ & $\begin{array}{l}n \\
\hat{0} \\
0 \\
+ \\
+\end{array}$ & $\begin{array}{l}0.921 \pm \\
0.069^{\mathrm{a}}\end{array}$ & $\begin{array}{l}\overrightarrow{\mathbb{N}} \\
\text { id } \\
+\end{array}$ \\
\hline \multicolumn{2}{|c|}{ F-probability } & \multicolumn{2}{|c|}{$\mathrm{P}<0.001$} & \multicolumn{2}{|c|}{$\mathrm{P}<0.001$} & \multicolumn{2}{|c|}{$\mathrm{P}<0.01$} & \multicolumn{2}{|c|}{$\mathrm{P}<0.0001$} & \multicolumn{2}{|c|}{$\mathrm{P}<0.05$} & \multicolumn{2}{|c|}{$\mathrm{P}<0.01$} \\
\hline \multirow{2}{*}{\multicolumn{2}{|c|}{ LSD at $5 \%$}} & \multicolumn{2}{|c|}{12.200} & \multirow{2}{*}{\multicolumn{2}{|c|}{$\frac{33.679}{45.933}$}} & \multicolumn{2}{|c|}{4.206} & \multicolumn{2}{|c|}{59.323} & \multicolumn{2}{|c|}{16.655} & \multicolumn{2}{|l|}{0.185} \\
\hline & & \multicolumn{2}{|c|}{16.639} & & & \multicolumn{2}{|c|}{5.737} & \multicolumn{2}{|c|}{80.908} & \multicolumn{2}{|c|}{22.715} & \multicolumn{2}{|l|}{0.252} \\
\hline
\end{tabular}

- Data are expressed as Mean \pm SE. Number of animals in each group is six.

- Means, which share the same superscript symbol(s), are not significantly different. 
Table 4: The effect of furfural on some kidney functions tests in control, low ,moderate and high dose groups

\begin{tabular}{|c|c|c|c|c|c|c|c|}
\hline \multicolumn{2}{|c|}{ Group } & $\begin{array}{l}\text { Uric acid } \\
(\mathrm{mg} / \mathrm{dl})\end{array}$ & $\%$ & $\begin{array}{c}\text { Creatinine } \\
\text { (mg/dl) }\end{array}$ & $\%$ & $\begin{array}{c}\text { Urea } \\
(\mathrm{mg} / \mathrm{dl})\end{array}$ & $\%$ \\
\hline \multicolumn{2}{|r|}{ Control } & $3.100 \pm 0.150^{\mathrm{a}}$ & & $1.091 \pm 0.059^{\mathrm{a}}$ & & $36.583 \pm 3.429^{b}$ & \\
\hline \multirow{3}{*}{ 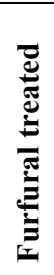 } & low dose & $3.550 \pm 0.117^{\mathrm{a}}$ & +14.516 & $1.100 \pm 0.031^{\mathrm{a}}$ & +0.917 & $48.533 \pm 2.890^{\mathrm{a}}$ & +32.665 \\
\hline & Moderate dose & $2.966 \pm 0.088^{a}$ & -4.322 & $1.183 \pm 0.134^{\mathrm{a}}$ & +8.432 & $57.400 \pm 2.818^{a}$ & +56.903 \\
\hline & high dose & $3.000 \pm 0.223^{\mathrm{a}}$ & -3.225 & $1.125 \pm 0.104^{\mathrm{a}}$ & +3.116 & $52.250 \pm 2.219^{a}$ & +42.820 \\
\hline \multicolumn{2}{|c|}{ F-probability } & \multicolumn{2}{|c|}{$\mathrm{P}>0.05$} & \multicolumn{2}{|c|}{$\mathrm{P}>0.05$} & \multicolumn{2}{|c|}{$\mathrm{P}<0.01$} \\
\hline \multicolumn{2}{|c|}{ LSD at $5 \%$} & \multicolumn{2}{|l|}{-} & \multicolumn{2}{|l|}{-} & \multicolumn{2}{|c|}{8.472} \\
\hline \multicolumn{2}{|c|}{ LSD at $1 \%$} & \multicolumn{2}{|l|}{-} & \multicolumn{2}{|l|}{-} & \multicolumn{2}{|c|}{11.554} \\
\hline
\end{tabular}

- Data are expressed as Mean \pm SE. Number of animals in each group is six.

- Means, which share the same superscript symbol(s), are not significantly different. 


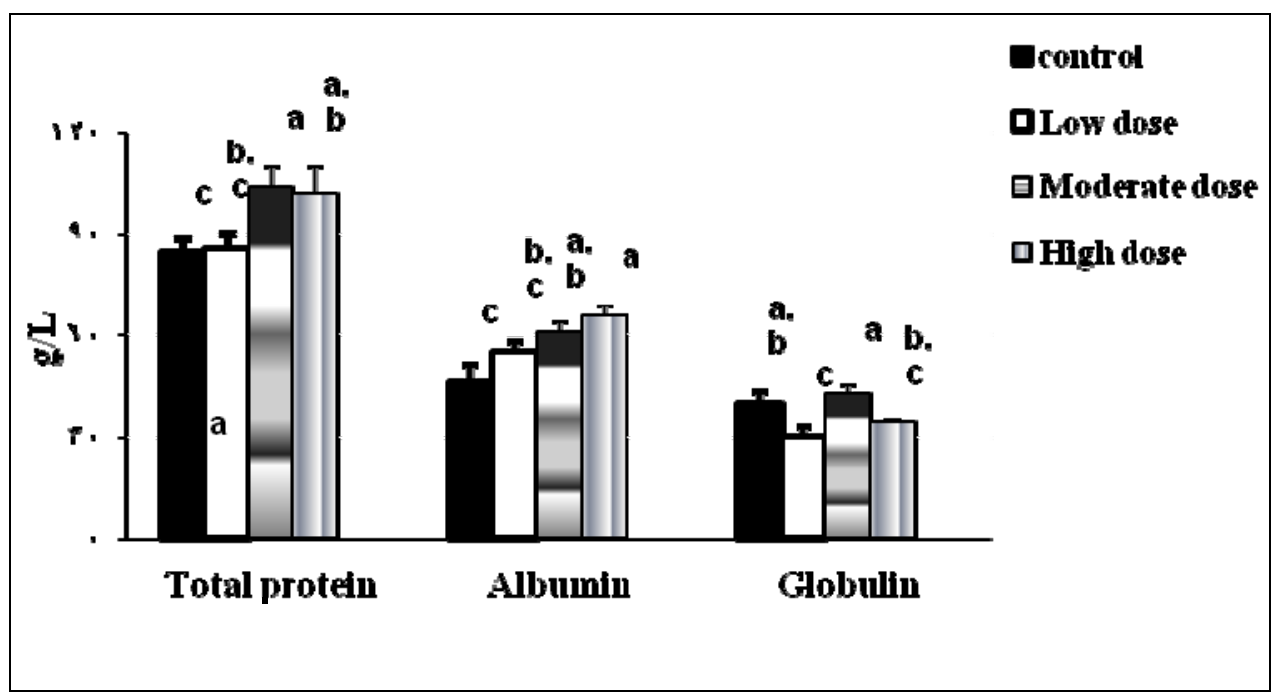

Figure 1: The effect of furfural on plasma protein levels in control, low ,moderate and high dose groups. Means, which share the same superscript symbol(s), are not significantly different. F-prob.: $\mathbf{P}<\mathbf{0 . 0 0 1}$.

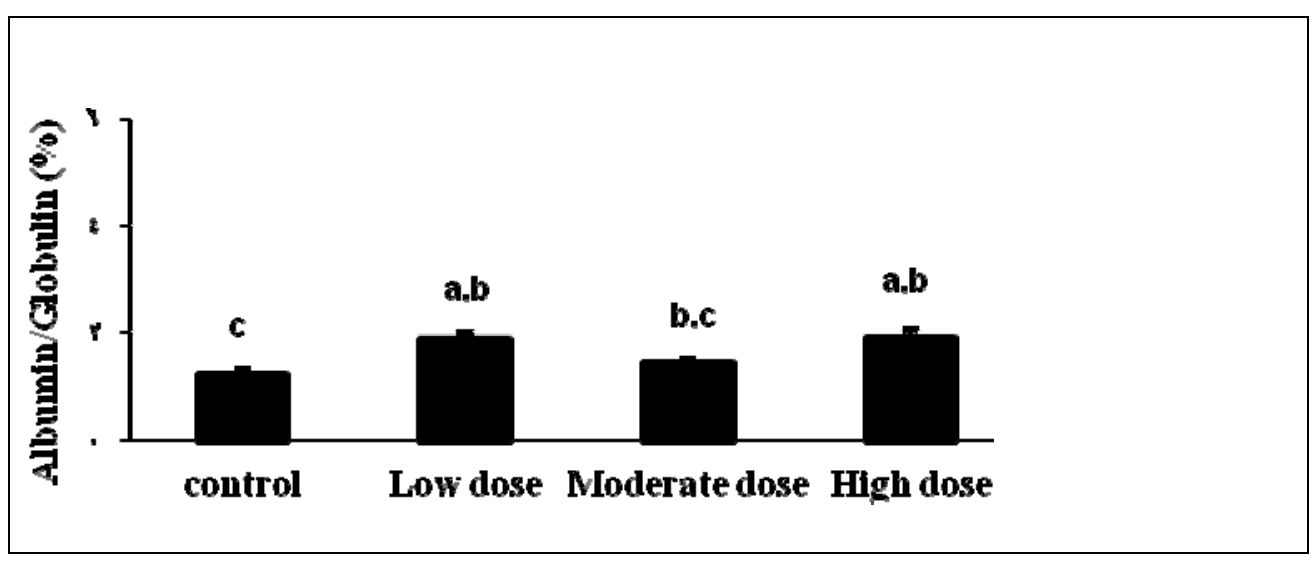

Figure 2: The effect of furfural on in albumin/globulin ratio (A/G ratio) in control, low, moderate and high dose groups. Means, which share the same superscript symbol(s), are not significantly different. F-prob.: $\mathbf{P}<\mathbf{0 . 0 0 1}$. 


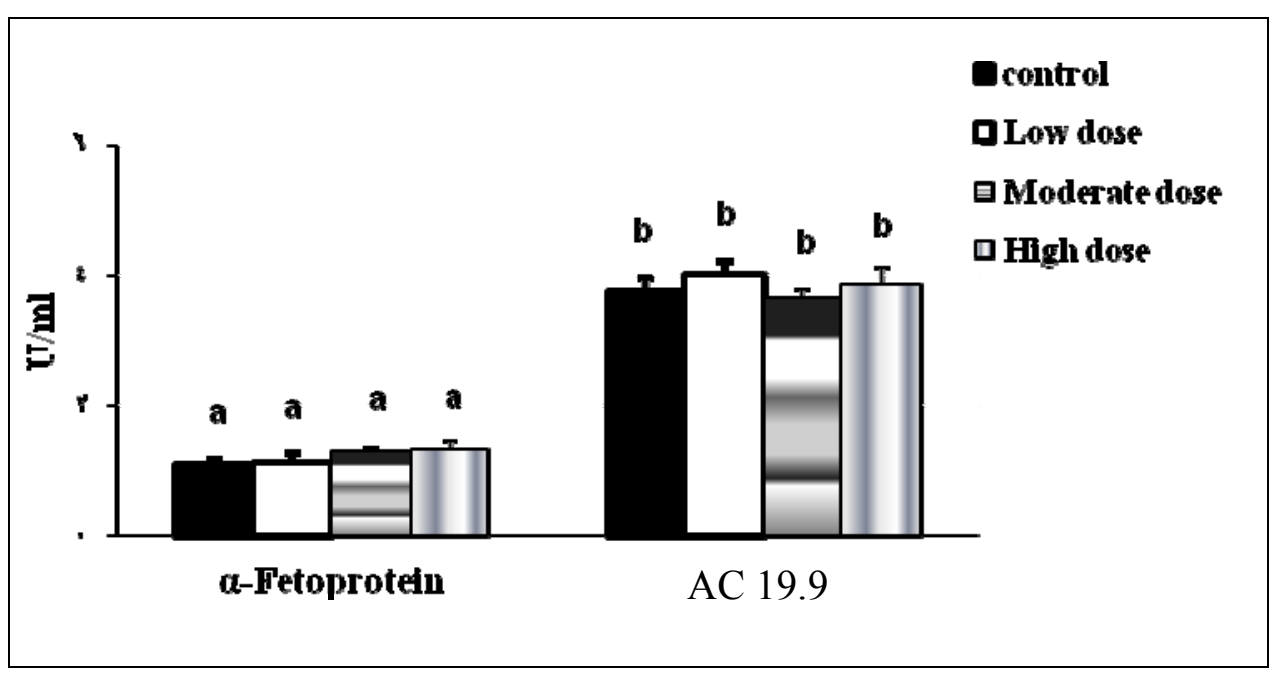

Figure 3:The effect of furfural on Serum $\alpha$-fetoprotein (AFP) and carbohydrate antigen (CA)-19.9 in control, low, moderate and high dose groups. Means, which share the same superscript symbol(s), are not significantly different. F-prob.: $\mathbf{P}<\mathbf{0 . 0 0 1}$.

\section{DISCUSSION}

Furfural has been classified as GRAS (Generally Recognized as Safe) by the Flavor Extract Manufacturers Association (FEMA) ${ }^{(\mathbf{1})}$. The substance 2-furancarboxaldehyde was identified as a high priority for assessment of human health risk because it was considered to present greatest potential for exposure (GPE) and had been classified by other agencies on the basis of carcinogenicity. ${ }^{(38,39)}$. The present study shows that the administration of furfural to albino rats for 8 weeks produced significant decrease of red blood corpuscles count, white blood cells (WBC), hematocrit (PCV) and hemoglobin content at the medium and high doses in a dose dependent manner. However, mean cell volume (MCV) of red blood corpuscles as well as mean cell hemoglobin (MCH) were highly significantly increased as a result of administration of medium and high concentration of furfural in a dose dependent manner. These results were consistent with the findings of Jonker (2000b) $)^{(40)}$ study on Fischer 344 rats were administered microencapsulated furfural via the diet for 13 weeks, there were some hematological changes as decrease in erythrocytes count in males in the highest dose group with increased cell volume and mean corpuscular hemoglobin in the top two dose groups males were observed. The effect may be attributed to effect of 
furfural on DNA of the erythropoietic cells which impairs the cell division. Furfural reacts with DNA in vitro, primarily at AT base pairs, leading to destabilization of the secondary structure of DNA and to single-strand breaks $^{(41,42,43)}$. Negative ${ }^{(44)}$ or weakly positive results ${ }^{(45)}$ have been obtained for most bacterial tests for genotoxicity. In particular, positive results were obtained in three out of several assays for reverse mutation in Salmonella typhimurium at relatively high concentrations in the absence of metabolic activation. It induced Sister chromatid exchange (SCE) in cultured Chinese hamster ovary (CHO) cells and human lymphocytes ${ }^{(46)}$.

The present study also revealed that, esinophils percentage was highly significantly depleted as a result of low and medium doses administration. This finding may be attributed to the infiltration of liver by esinophils as shown by the study of Shimizu and Kanisawa $^{(9,12)}$ who evidenced the presence of liver damage in the form of scattered esinophilic globular formation and increase mitotic figures without zonal or massive necrosis observed 6 hours after exposure to furfural by gavage. Jonker ${ }^{(40,47)}$ in their study, found changes in the perilobular region in $5 / 10$ of all studied rats ,mainly, including cells with less coarse cytoplasm and increased clumping of esinophils ${ }^{(40,47)}$.

The liver synthesizes enzymes and structural proteins, detoxifies many internal and external products of the organism ${ }^{(48)}$. For these functions, hepatocytes use enzyme systems. Furfural is not harmful. However, its by-product, pyromucic acid, has detrimental effect ${ }^{(49)}$. The present study revealed an increase in the liver enzymes, total bilirubin concentration as well as plasma albumin and albumin/globulin ratio. Jonker ${ }^{(40)}$ study on Fischer 344 rats that were administered microencapsulated furfural for 13 weeks revealed, in females, a decrease in serum alkaline phosphatase, an increase in gamma glutamyl transferase and an increase in plasma albumin in the highest dose group. In males in the high dose group there was a decrease in ALT, an increase in plasma albumin and albumin/globulin ratio. The increase in liver enzymes as a result of furfural administration could be explained by inflammation and tissue damage induced by furfural effect on the liver hepatocytes.

Irwin ${ }^{(50)}$ in a subchronic toxicity study revealed that furfural causes centrilobular necrosis and multifocal subchronic inflammation of the liver were observed in males at $150 \mathrm{mg} / \mathrm{kg}$ body weight per day, only, and at 300 $\mathrm{mg} / \mathrm{kg}$-body weight per day, the same liver effects were observed both in males and females .

The increase in serum total protein and albumin levels as a result of medium and high doses of furfural in the present study may be explained on the basis liver cirrhosis induced by furfural.

In rat cirrhotic liver, there were no significant differences in levels of serum albumin or albumin mRNA expression between cirrhotic and normal liver. In primary hepatocyte culture, albumin mRNA expression, the amount of albumin secretion and the albumin promoter activity were clearly enhanced in cirrhotic 
hepatocytes compared to normal hepatocytes ${ }^{(14)}$.

Serum globulin level, on the other hand, was significantly elevated due only to low dose. This finding was in agreement with the results of the study of Agakishiev et al. (51) which revealed that application of furfural resulted in imbalanced levels of $\operatorname{IgG} 1, \operatorname{IgG} 2, \operatorname{Ig} \mathrm{A}$, and $\operatorname{IgM}$ after exposure of guinea pig skin to furfural.

The use of tumor markers has become a very attractive method for the detection and diagnosis of neoplastic diseases ${ }^{(\mathbf{5 2 , 5 3 )}}$. However, their value in cancer detection has been controversial largely because no single tumor marker is sensitive and specific enough to meet strict diagnostic criteria $^{(54)}$. Attallah et

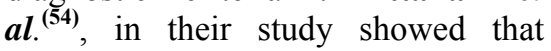
$\mathrm{CA}_{19.9}$ has the best sensitivity for pancreatic cancer. In hepatocellular carcinoma, AFP was the most sensitive tumor marker. The mode of action underlying the hepatocarcinogenic activity of furfural after oral exposure has not fully been elucidated. However, a genotoxic component clearly is not involved, as evidenced by the in vivo test using transgenic animals. The data do, however, point to a possible role for chronic cytotoxicity that is found in conjunction with the induction of tumors; a pathway that has also been accepted for other non-genotoxic hepatocarcinogens.

In the present study, we investigated the levels of serum AFP and CA 19.9 as markers for early detection of hepatocellular carcinoma but these levels were non-significantly affected as a result of all tested doses of furfural. In our study, the serum urea level was highly significantly increased as a result of all tested doses of furfural; The urea/creatinine ratio was only significantly decreased as a result of medium and high doses. However, in the study of Jonker ${ }^{(55)}$, female rats at some doses had decreased blood urea nitrogen and creatinine concentrations, but these changes were not dose-related. This may be due to the effect of furfural on the kidney when it is eliminated through it.

\section{CONCLUSIONS}

The administration of furfural to albino rats for 8 weeks produced macrocytic anemia, leucopenia, elevated liver enzymes and bilirubin indicating hepatic damage. Mainly these changes are dose related, but there is no evidence of carcinogenic effect by tumor markers.

\section{REFERENCES}

1. Adams TB, Doull J, Goodman JI, Munro IC, Newberne P, Portoghese PS, Smith RL, Wagner BM, Weil CS, Woods LA, Ford RA.(1997): The FEMA GRAS assessment of furfural used as a flavor ingredient. Flavor and Extract Manufacturer's Association. Food Chem. Toxicol., 35(8):739-51.

2. Begic-Janeva AZ (1991): Patologija jetre, zucne besike i zucnih vodova. Decje novine, Gornji Milanovac, 1991.

3. Blitzer BL, Boyer JL (1982): Cellular mechanisms of bile 
formation. Gastroenterology 82: 346-57.

4. Higashi T.(1937): On some constituents of Sake. Sci Pap Inst Phys Chem Res.,33: 1-129.

5. Petković M. Hemija biomolekula III, Univerzitet u Nišu, 1990.

6. Burdock G. 2010. Fenaroli's handbook of flavor ingredients. 6th ed. Boca Raton (FL): CRC Press. Furfural. p. 705-706.

7. NTP, 1990a. NTP technical report on the toxicology and carcinogenesis studies of furfural (CAS no.98-01-1) in $\mathrm{F} 344 / \mathrm{N}$ rats and B6C3F1 mice (gavage studies). March 1990. NTP-TR 382. NIH Publication no. 902837.

8. Maruyama H, Amanuma $T$, Takashima Y, Yoshiji H Nakae D, Tsutsumi M, Tsujiuchi T, Denda A, Konishi Y .(1992): Possible enhancing effect of the immunosuppressive agent, 6 mercaptopurine(6-MP) on focal lesion development in cirrhotic liver induced by carbon tetrachloride but not furfural in F344 rats. Carcinogenesis 1992 13(8): 1365-9

9. Shimizu A, Kanisawa M.(1986): Experimental studies on hepatic cirrhosis and hepatocarcinogenesis. Production of hepatic cirrhosis by furfural administration. Acta Pathol. Jpn., 36(7): 1027-1028.

10. Mishra A.(1992): Furfural: a toxic chemical. Agric. Biol. Res., 1992; 8: 93-104

11. Jonek J, Konecki J, Kaminski M.(1975): Histoenzymatic changes in liver in acute poisoning with furfural. Morphol. Embryol., 21(1): 47-51.

12. Kaminska O, Gruszecka B.(1977): Dynamics of morphological and histochemical changes in rats' liver in chronic furfural poisoning. Medycyna Pracy 28(5): 377-391.

13. Kiso S, Kawata S, Tamura S, Ito N, Takaishi K, Shirai $\mathbf{Y}$ (1994): Alteration in growth regulation of hepatocytes in primary culture obtained from cirrhotic rat: poor response to transforming growth factor- $\beta 1$ and interferons. Hepatology 20(5): 1303-1308.

14. Koura T, Kaneko S, Matsushita E, Ohno H, Kaji K, Kobayashi K(1999): Investigation of albumin-synthesizing ability in rat cirrhotic liver-derived hepatocytes using primary hepatocyte culture. J. Hepatology 31: 293-299.

15. Ballmer PE, Walshe D, McNurlan MA, Watson H, Brunt PW, Garlick PJ. Albumin synthesis rates in cirrhosis: correlation with Child-Turcotte classification. Hepatology 1993; 18(2): 292-297.

16. Divald A, Jeney A, Nagy OJ, Timar F, Lapis K(1990): Modification of the inhibitory effects of $\mathrm{CCl}_{4}$ on phospholipid and protein biosynthesis by prostacyclin. Biochem. Phamacol., 40(70): 1477-83.

17. Ernest D. Olfert, DVM; Brenda M. Cross, DVM; and A. Ann McWilliam. Guide To the Care and Use of Experimental Animals. CCAC, Canada, Vol. 1, 1-298. 
18. Jenner, P.M., Hagan, E.C., Taylor, J.M., Cook, E.L., Fitzhugh, O.G., 1964. Food flavourings and compounds of related structure. I.Acute oral toxicity. Food and Cosmetics Toxicology 2, 327-343.

19. Wintrobe, N. M.; Lee, G. R.; Boggo, D. R.; Bithell, T. G.; Forester, J. Athens, J.W. and Lukens, J. N. (1981): Clinical Haematology. $8^{\text {th }}$ Edition. Lea and Febiger, Philadelphia, USA, pp. 255-285.

20. Miale, J.B. (1972): Laboratory Medicine: Haematology. $4^{\text {th }}$ Edition. The C. V. Mosby Co., Saint Lucis, pp. 1200-1210.

21. Dacie, S.J. and Lewis, S.M. (1991): Practical Haematology. 7th Edition. Churchill, Livingstone.

22. Lucky, Z. (1977): Methods of diagnosis of fish disease. Amm. Publication PVLtd, New Delhi, Bombay, Calcutta and New York, pp. 345-351.

23. Houwen B. (2000): Blood film preparation and staining procedures. Laboratory Hematology 6:1-7.

24. Bergmeyer, H.U.; Scheibe, P. and Wahlefeld, A.W. (1978). Optimization of methods for aspartate aminotransferase and alanine aminotransferase. Clin. Chem., 24: 58-73.

25. Beleta, J. and Gella, F. J. (1990). Metodo recomendado para la determinacion en rutina de la concentracion catalitica de la gamma-glutamyltransferasa en sero sanguineo humano/ Quim Clin 9: 58-61.
26. Belfield A and Goldberg DM (1971). Colorimetric determination of alkaline phosphates activity. Enzyme 12: 561-568.

27. Buhl SN, Jackson KY (1978). Optical conditions and comparisons of lactate dehydrogenase catalysis of the lactate-to-pyruvate and pyruvateto-lactate reactions in human serum at 25,30 and $37{ }^{\circ} \mathrm{C}$. Clin. Chem., 24(5): 828-831.

28. Jendrassik L, Grap P. (1938). Determination of bilirubin by enzymatic colorimetric method. Biochem. Z, 297: 81-89.

29. Henry, R.J. (1964). Clinical Chemistry. Harper and Row publisher, New York. P. 181

30. 30-Doumas BT, Watson WA, Biggs HG (1971). Albumin standards and the measurement of serum albumin with bromcresol green. Clin. Chim. Acta 31: 8796.

31. Waldmann, T.A. and Mclntire, K.R. (1974). The use of radioimmunoassay for alphafetoprotein in the diagnosis of malignancy. Cancer, 34: 15101505.

32. Wepsic, H.T. (1981). Alphafetoprotein: its quantitation and relationship to neoplastic disease. In: Alpha-fetoprotein: "Laboratory Procedures and Clinical Applications". Krikpatrik, A.M. and Nakamura, R.M. (eds). New York:Masson publishing. p 115-129.

33. Frebourg $T$, Bercoff $E$, Manchon N, Senant J, Basuyau JP, Breton P, Janvresse A, Brunelle P, Bourreille J.(1988). 
The evaluation of $\mathrm{CA}_{19.9}$ antigen level in the early detection of pancreatic cancer: A prospective study of 866 patients. Cancer 62(11): 2287-90.

34. Patton, C.J. and Crouch, S.R. (1977): Spectrophotometric and kinetics investigation of the Berthelot reaction for the determination of urea. Anal. Chem., 49: 464469.

35. Henry, R.J. (1974): Clinical Chemistry, Principles and Techniques, $2^{\text {nd }}$ edition, Harper and Row, pp: 525.

36. Barham, $D$. and $P$. Trinder. (1972): Enzymatic determination of uric acid. Analyst 97: 142-145.

37. PC-STAT (1985): One-way analysis of variance. Version $1 \mathrm{~A}$ (C) copyright. The University of Georgia. Programs coded by Rao, M.; Blane, K. and Zonneberg, M. University of Georgia, USA.

38. Canada, Dept. of the Environment, Dept. of Health. 2009a.Canadian Environmental Protection Act, 1999: Notice of eleventh release of technical information relevant to substances identified in the Challenge. Canada Gazette, Part I, Vol. 143, No. 39

39. Canada, Dept. of the Environment.

(2009b): Canadian

Environmental Protection Act, 1999: Notice with respect to Batch $11 \quad$ Challenge substances. Canada Gazette, Part I, Vol. 143, No.39.

40. Jonker, D. (2000b): Amendment 1 to the TNO-report V99.520: Subchronic (13-week) oral toxicity study in rats with microencapsulated furfural. Unpublished report from TNO, Zeist, Netherlands, Submitted to WHO by The Flavour and Extract Manufacturers' Association of the United States.

41. Uddin S (1993): Effect of furfural on the secondary structure of DNA. Med. Sci. Res., 21: 545-546.

42. Uddin S, Rahman H, Hadi SM (1991): Reaction of furfural and methylfurfural with DNA: use of single-strand specific nucleases. Food Chem. Toxicol. ,29(10): 719-721.

43. Hadi SM, Uddin S, Rehman A (1989). Specificity of the interaction of furfural with DNA. Mutat. Res. 225(3): 101-106.

44. Aeschbacher, H.U., Chappuis, C., Manganel, M. \& Aeschbach, R. (1981): Investigation of Maillard products in bacterial mutagenicity test systems. Prog. Food. Nutr. Sci., 5:279-293.

45. Loquet, C., Toussaint, G. \& LeTalaer, J.Y. (1981): Studies on mutagenic constituents of apple brandy and various alcoholic beverages collected in Western France, a high incidence area for esophageal cancer. Mutation Res., 88: 155-164.

46. Galloway, S.M., Bloom, A.D., Resnick, M., Margolin, B.H., Nakamura, F., Archer, P. \& Zeiger, E., 1985. Development of standard protocol for in vitro cytogenetic testing with Chinese hamster ovary cells: comparison of results for 22 compounds in two laboratories. Environmental Mutagenesis 7(1): 1- 51. 
47. Jonker, D. (2000c): Sub-chronic (13-week) oral toxicity study in rats with micro-encapsulated furfural. Unpublished report V99.520 from TNO, Zeist, Netherlands. Submitted to WHO by The Flavour and Extract Manufacturers' Association of the United States

48. Parkash KK, Caldvell J (1994): Metabolism and excretion of $\mathrm{S} 12 \mathrm{Cc}$ furfural in the rat and mouse. Food. Chem. Toxicol.,; 32(10): 887-95.

49. Draper AJ, Madan A, Parkinson A.(1997): Inhibition of coumarin 7-hydroxylase activity in human liver microsomes. Arch. Biochem. Biophys., 341(1): 47-61

50. Irwin R. (1990): NTP technical report on the toxicology and carcinogenesis studies of furfural in $\mathrm{F} 344 / \mathrm{N}$ rats and $\mathrm{B} 6 \mathrm{C} 3 \mathrm{~F} 1$ mice (gavage studies). Research Triangle Park, NC 27709

51. Agakishiev DD, Guzova VA, Batalova TN, Afanas'ev SS. (1990): The level of immunoglobulins in the blood serum of guinea pigs with epicutaneous exposure to petroleum refinery products]. Vestn Dermatol Venerol 8: 29-32 (1990)

52. Pectasides, D., A. Mylonakis, M. Kostopoulou, M.
Papadopoulou and D. Triantafillis, Varthalitis J, Dimitriades M, Athanassiou A.(1997): CEA, CA 19-9 and CA-50 in monitoring gastric carcinoma. Am. J. Clin. Oncol., 20: 348-353.

53. Yamao, T., S. Kai, A. Kazami, K. Koizumi, T. Handa, N. Takemoto and M. Maruyama, (1999): Tumor markers CEA, CA 19-9 and CA 125 in monitoring of response to systemic chemotherapy in patients with advanced gastric cancer. Jpn. J. Clin. Oncol., 29: 550-555.

54. Attallah A.M., N.A. AlGhawalby, A.A.F. Abdel Aziz, E.A. El-Sayed, A.A. Tabll and A.M. El-Waseef , (2006): Clinical Value of Serum CEA, CA 19-9, CA 242 and AFP in Diagnosis of Gastrointestinal Tract Cancers. International Journal of Cancer Research, 2: 50-56.

55. Jonker, D. (2000a): Dose range finding study (14-day) with micro-encapsulated furfural in F344 rats. Unpublished report V98.1173 from TNO, Zeist, Netherlands. Submitted to WHO by The Flavour and Extract Manufacturers' Association of the United

States. 


\section{تأثير مادة الفورفورال كنكهة طعام على الجوانب الدموية والبيوكيميائية في

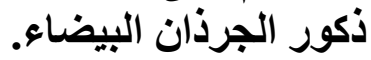

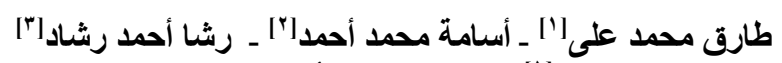

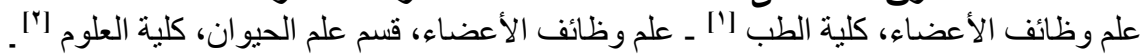

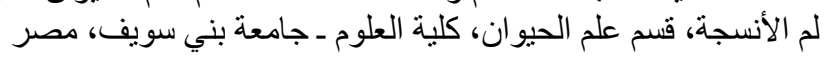

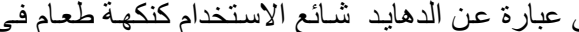

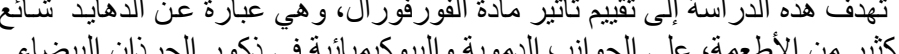
المواد وألاساليب الاطعة الميب

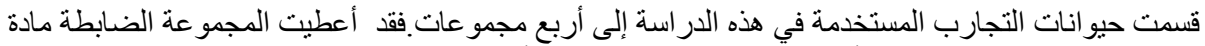

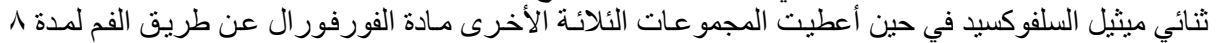

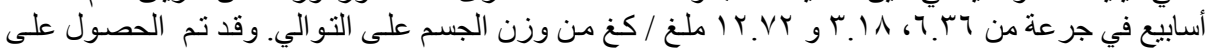

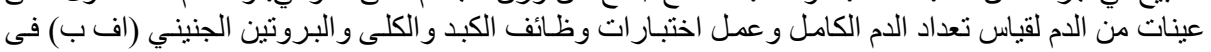

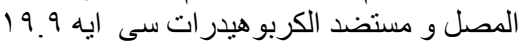
النتائج

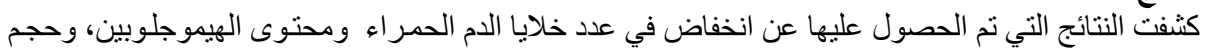

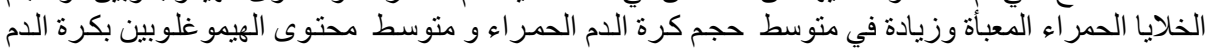

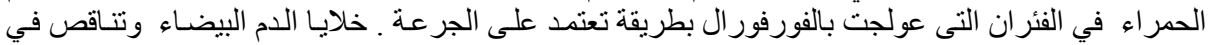

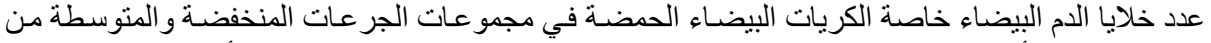

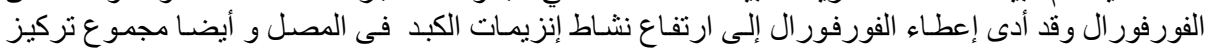

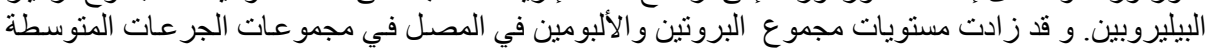

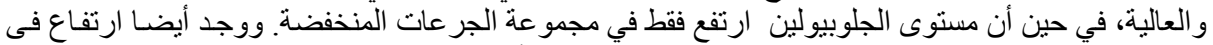

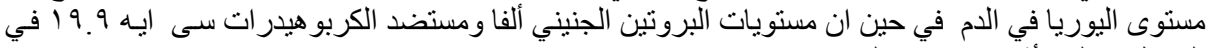

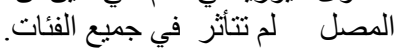
الاستنتاجات

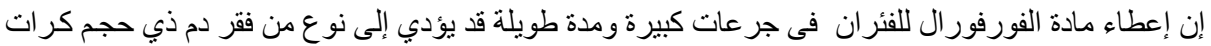

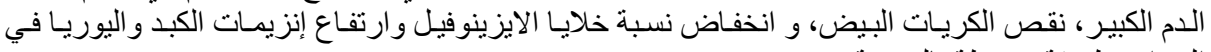
الفئر ان بطريقة مرتبطة بالجر عنة الكربة 